http://jmscr.igmpublication.org/home/ ISSN (e)-2347-176x ISSN (p) 2455-0450 crossref DOI: https://dx.doi.org/10.18535/jmscr/v7i8.133

Journal Of Medical Science And Clinical Research

\title{
Outcome of cow dung powder poisoning depending upon clinical profile- Retrospective study
}

\author{
Authors \\ Dr M.N. Shamala\#. MD. AP, Dr S.Senthil Nathan*. MD. Associ. Prof. \\ Prof. Dr R.Ramasamy.MD, Professor (HOD). R.Anbalagan.MD
}

Department of General Medicine, Annapoorna Medical College \& Hospital, Salem, Tamilnadu, India *Corresponding Author

Dr S.Senthil Nathan. MD. Associ. Prof.

\begin{abstract}
Background and Objective: Poisoning is an important health problem causing significant morbidity and mortality throughout the world. The general pattern of poisoning in a particular region will help in early diagnosis and treatment of cases, thus decreasing the rate of mortality and morbidity.

The present study was carried out with the objective to investigate the pattern of acute Cow Dung Powder Poisoning (Auramine-O, yellow dye) in a tertiary care hospital, Salem DT. Tamil Nadu, India.

Material and Method: A retrospective analysis of all Cow dung powder poisoning (yellow -dye Auromine-O) admitted in the department of Annapoorna Medical Collage \& Hospital. Salem, Tamil Nadu, India. From January 2014 to July 2019 was done to study the Out-Come of Cow dung powder poisoning. Data regarding Age, Sex, marital status, occupation, religion, locality, rout of exposure, time elapsed after intake. Circumstances of poisoning, duration of hospital Admission and outcome were collected and analyzed.

Conclusion: This study adds information to the existing data which may help to develop prevention statergies. Health education to the house wives and coolies and student by either by house trained faculty or phsycologists and early detection of risk taking behavior in adults to some extent to prevent self harming in all patients

Keyword: Cow dung powder' Saani Powder', Yellow Powder (Auramine-O Poison).
\end{abstract}

\section{Introduction}

Cow dung powder poisoning is more common in surrounding village of salem district. It was used as suicidal poisoning, self poisoning in south India, Tamilnadu. Knowledge of General pattern of poisoning in a particular region will help in early diagnosis and treatment of cases, then decreasing rate of mortality morbidity. Information available in our locality with regard to cow dung powder poison is limited. The present study was came out with the object to investigate the pattern of cow dung powder poisoning in a tertiary care hospitals which includes the popular demographic distribution of cases depending on the nature of poison and treatment.

\section{Material and Methods}

This is a retrospective record based study conducted in a tertiary care hospital. The study included all diagnosed case of yellow cow dung powder poisoning at all age groups reported during 5and1/2yrs time period between January 
2014 to July 2019.The study was conducted with the approval from Institute Ethics committee and confidentiality of data was ensured.

Data regarding age, sex, marital status, occupation, religion ,locality, route of exposure, time lapsed after intake, circumstances of poisoning, name of the poison, chemical type, duration of hospitalization and outcome were collected was entered in computer data base and analysed by using proportion and Chi-square.

\section{Results}

In the present study 29 cases of poisoning were reviewed retrospectively. All reported cases were found to be suicidal poisoning. In all cases the roue of exposure was oral. Females $(16,55.2 \%)$ outnumbered Males ( $13,44.8 \%)$. Majority of cases were

\section{AGE GROUP}

\begin{tabular}{|c|c|c|c|c|c|}
\hline \multicolumn{2}{|c|}{} & Frequency & Percent & $\begin{array}{c}\text { Valid } \\
\text { Percent }\end{array}$ & $\begin{array}{c}\text { Cumulative } \\
\text { Percent }\end{array}$ \\
\hline \multirow{4}{*}{ Valid } & $15-20$ & 8 & 27.6 & 27.6 & 27.6 \\
\cline { 2 - 6 } & $21-30$ & 13 & 44.8 & 44.8 & 72.4 \\
\cline { 2 - 6 } & $31-40$ & 6 & 20.7 & 20.7 & 93.1 \\
\cline { 2 - 6 } & $41-50$ & 2 & 6.9 & 6.9 & 100.0 \\
\cline { 2 - 6 } & Total & 29 & 100.0 & 100.0 & \\
\hline
\end{tabular}

In age group (19-50yrs)

Most of the patients hospitalized within six hours after toxic exposure. No previous historyof poisoning is seen in all. Gastric lavage was done for all the cases. Specific antidote is not available. Treated symptomatically. All patients received adequate symptomatic and supportive life saving treatment in the form of mechanical ventilation .no intubation in our study, no mortality or death recorded in our study.

\section{Discussion}

The present study showed that the cow dung powder poisoning data collected were from Villages surrounding our Annapoorna Medical College \& Hospital, siragapadi, salem DT T.N.INDIA. Majority of cases were from village ellampillae nearest distance to the hospital.
Cow dung powder poison available in two different colours, yellow powder (Aromine-o) And green powder (Malachte green) commoner in rural Tamilnadu (south India) in the District as Coimbatore, Erode and Tripura. Even though powder sale is legally banned the powder is easily available in grocery shops. It can cause gastrointestinal symptoms and persistent seizure some times.

Cow dung powder poisoning was found to be most common poisoning consumed in our surrounding localities. It has aptly said by various researchers that the pattern of poisoning in a region depends upon various factors such as availability, cost and access to toxic agents, Socioeconomic status, cultural and religious characteristic to people.

It is interesting to note that this powder has been so widely used, that the district authority banned sales of the powder in 2007. However it is still widely available and there is no trend of a decrease in the incidence of cow dung powder poisoning during the study period.

This under scarce the fact that banning such substance without educating the public or tacklin16;g fundamental cause for self harming will not succeed. The present study showed that Cow dung powder is more common in females compared to Males in this locality. The male to female ratio was (1:2) . This pattern was observed in our study. Poisoning in students in the locality was equal in both sexes reflect their mental vulnerability to stress in the form as Failure in the exam, Maladjustment and inability to cope up with the high expectation from patients. Higher degree of stress in academic, financial and social sectors as well as inability to achive the target on professional, educational and socio economic forms leading to limited altrations could be the contributory factor in taking suicidal act.

The house wives were the next vulnerable groups as they are easily exposed to the poisoning agents, particularly Cow dung powder poison.

Factors like Dowry, cruelty by in-laws, family quarrels, mal adjustment in marriage life and 
dependency of women and house band and responsible for the higher incidence of poisoning among house wives.

Most of the patent in this study were in the young age group and maximum number of pts $(29,100 \%)$ was age group of 19-20years followed by $(8,27.6$ $\%$ ) in 21-30 yr age groups. since all the cases were suicidal intention the distribution pattern shows the mental vulnerlabity and impulsiveness of our patients., loss, abuse, mental illness and pressure from cultural and social backgrounds could be the possible risk factor. studies in the past revealed that poisoning was more common in the age group 20-30 yrs. the change noted in this study can be attributed to the fact that the people are subjected to substantial amount of mental stress much earlier in their life in terms of adapting to modern life styles, failure in love, family problems.

In our study, overall mortality was found to be $(0 \%)$. there was no mortality in our present study. in our locality cow dung powder poisoning more common. In our study the patients consumption of powder was in small quantity. Not lethal dose. The approach to emergency ward was earlier to $1 / 2$ an hour to maximum hours of 3-5hours.

Immediate treatment and follow up and management resulted in less mortality. Patient has only Gastro intestinal irritation and few episodes of vomiting, all other parameters were normal. Incidence maximum in the age group 21-30 yrs, $(13,44.8 \%)$, minimum age group were 41-50 $(2,6.9 \%)$.

\section{AGE GROUP}

\begin{tabular}{|c|c|c|c|c|c|}
\hline \multicolumn{2}{|c|}{} & Frequency & Percent & $\begin{array}{c}\text { Valid } \\
\text { Percent }\end{array}$ & $\begin{array}{c}\text { Cumulative } \\
\text { Percent }\end{array}$ \\
\hline \multirow{5}{*}{ Valid } & $15-20$ & 8 & 27.6 & 27.6 & 27.6 \\
\cline { 2 - 6 } & $\begin{array}{c}21- \\
30\end{array}$ & 13 & 44.8 & 44.8 & 72.4 \\
\cline { 2 - 6 } & $31-40$ & 6 & 20.7 & 20.7 & 93.1 \\
\cline { 2 - 6 } & $41-50$ & 2 & 6.9 & 6.9 & 100.0 \\
\cline { 2 - 6 } & Total & 29 & 100.0 & 100.0 & \\
\hline
\end{tabular}

Attempt were more in female $(16,55.2 \%)$ than males $(13,44.8 \%)$

\begin{tabular}{|c|c|c|c|c|c|}
\hline \multicolumn{6}{|l|}{ SEX } \\
\hline & & Frequency & Percent & $\begin{array}{c}\text { Valid } \\
\text { Percent }\end{array}$ & $\begin{array}{l}\text { Cumulative } \\
\text { Percent }\end{array}$ \\
\hline \multirow{3}{*}{ Valid } & FEMALE & 16 & 55.2 & 55.2 & 55.2 \\
\hline & MALE & 13 & 44.8 & 44.8 & 100.0 \\
\hline & Total & 29 & 100.0 & 100.0 & \\
\hline \multicolumn{6}{|c|}{$\begin{array}{l}\text { All cases from nearest villages surrounding } \\
\text { villages from our collage hospital. }\end{array}$} \\
\hline \multicolumn{6}{|c|}{ DISTRICT } \\
\hline & & Frequency & Percent & $\begin{array}{c}\text { Valid } \\
\text { Percent }\end{array}$ & $\begin{array}{c}\text { Cumulative } \\
\text { Percent }\end{array}$ \\
\hline Valid & SALEM & 29 & 100.0 & 100.0 & 100.0 \\
\hline
\end{tabular}

By occupation maximum were house wives $(13,44.8 \%)$, followed by coolies $(10,34.5 \%)$.

\begin{tabular}{|c|c|c|c|c|c|}
\hline \multicolumn{2}{|c|}{ OCCUPATION } & Frequency & Percent & $\begin{array}{c}\text { Valid } \\
\text { Percent }\end{array}$ & $\begin{array}{c}\text { Cumulative } \\
\text { Percent }\end{array}$ \\
\hline \multirow{5}{*}{ Valid } & Coolie & 10 & 34.5 & 34.5 & 34.5 \\
\cline { 2 - 6 } & driver & 1 & 3.4 & 3.4 & 37.9 \\
\cline { 2 - 6 } & Driver & 1 & 3.4 & 3.4 & 41.4 \\
\cline { 2 - 6 } & HW & 13 & 44.8 & 44.8 & 86.2 \\
\cline { 2 - 6 } & Student & 4 & 13.8 & 13.8 & 100.0 \\
\cline { 2 - 6 } & Total & 29 & 100.0 & 100.0 & \\
\hline
\end{tabular}

Among religions Hindu (26, 89.7\%) followed by muslims $(2,6.9 \%)$ and Christians $(1,3.4 \%)$

\begin{tabular}{|c|c|c|c|c|c|}
\hline \multicolumn{2}{|l|}{ RELIGION } & Frequency & Percent & $\begin{array}{c}\text { Valid } \\
\text { Percent }\end{array}$ & $\begin{array}{c}\text { Cumulative } \\
\text { Percent }\end{array}$ \\
\hline \multirow{4}{*}{\begin{tabular}{l} 
Valid \\
\cline { 2 - 6 }
\end{tabular}} & Christian & 1 & 3.4 & 3.4 & 3.4 \\
\cline { 2 - 6 } & Hindu & 26 & 89.7 & 89.7 & 93.1 \\
\cline { 2 - 6 } & Muslim & 2 & 6.9 & 6.9 & 100.0 \\
\cline { 2 - 6 } & Total & 29 & 100.0 & 100.0 & \\
\hline
\end{tabular}

The time gap between consumption of poison to starting treatment was earliest was $0.5 \mathrm{hrs}(3.4 \%)$ to maximum of 9 hrs.(3.4\%),no of cases reaching to hospital $0.5 \mathrm{hrs}$ to $1 \mathrm{hr}$ was $8.1 .15-2 \mathrm{hrs}$, 6case, 2.15hrs-3hrs 9 cases,5to 6 hrs were 5 cases,,9hrs was 1 case, and signs staining of skin(1case had staining of lips. 


\begin{tabular}{|c|c|c|c|c|c|}
\hline & & Frequency & Percent & $\begin{array}{c}\text { Valid } \\
\text { Percent }\end{array}$ & $\begin{array}{c}\text { Cumulative } \\
\text { Percent }\end{array}$ \\
\hline \multirow{17}{*}{ Valid } & 0.25 & 1 & 3.4 & 3.4 & 3.4 \\
\hline & 0.5 & 2 & 6.9 & 6.9 & 10.3 \\
\hline & 1 & 4 & 13.8 & 13.8 & 24.1 \\
\hline & 1.149 & 1 & 3.4 & 3.4 & 27.6 \\
\hline & 1.3 & 1 & 3.4 & 3.4 & 31.0 \\
\hline & 1.5 & 1 & 3.4 & 3.4 & 34.5 \\
\hline & 2 & 3 & 10.3 & 10.3 & 44.8 \\
\hline & 2.15 & 1 & 3.4 & 3.4 & 48.3 \\
\hline & 2.299 & 1 & 3.4 & 3.4 & 51.7 \\
\hline & 2.30 & 2 & 6.9 & 6.9 & 58.6 \\
\hline & 3 & 5 & 17.2 & 17.2 & 75.9 \\
\hline & 3.3 & 1 & 3.4 & 3.4 & 79.3 \\
\hline & 5 & 2 & 6.9 & 6.9 & 86.2 \\
\hline & 5.30 & 2 & 6.9 & 6.9 & 93.1 \\
\hline & 6 & 1 & 3.4 & 3.4 & 96.6 \\
\hline & 9 & 1 & 3.4 & 3.4 & 100.0 \\
\hline & Total & 29 & 100.0 & 100.0 & \\
\hline
\end{tabular}

\begin{tabular}{|l|c|c|c|c|c|}
\hline \multicolumn{2}{|l|}{ STAINING LIPS } \\
\hline Other & Frequency & Percent & $\begin{array}{c}\text { Valid } \\
\text { Percent }\end{array}$ & $\begin{array}{c}\text { Cumulative } \\
\text { Percent }\end{array}$ \\
\hline \multirow{4}{*}{ Valid } & Lips & 1 & 3.4 & 3.4 & 3.4 \\
\cline { 2 - 6 } & Nil & 28 & 96.6 & 96.6 & 100.0 \\
\cline { 2 - 6 } & Total & 29 & 100.0 & 100.0 & \\
\hline
\end{tabular}

\begin{tabular}{|l|c|c|c|c|c|}
\hline \multicolumn{2}{|l|}{ GIT } & Frequency & Percent & $\begin{array}{c}\text { Valid } \\
\text { Percent }\end{array}$ & $\begin{array}{c}\text { Cumulative } \\
\text { Percent }\end{array}$ \\
\hline Valid & No & 29 & 100.0 & 100.0 & 100.0 \\
\hline
\end{tabular}

\begin{tabular}{|c|c|c|c|c|c|}
\hline \multicolumn{6}{|c|}{ EPIPAIN } \\
\hline & & Frequency & Percent & $\begin{array}{c}\text { Valid } \\
\text { Percent }\end{array}$ & $\begin{array}{c}\text { Cumulative } \\
\text { Percent }\end{array}$ \\
\hline \multirow{3}{*}{ Valid } & No & 26 & 89.7 & 89.7 & 89.7 \\
\hline & Yes & 3 & 10.3 & 10.3 & 100.0 \\
\hline & Total & 29 & 100.0 & 100.0 & \\
\hline \multicolumn{6}{|c|}{ NO. OF DAYS STAY } \\
\hline & & Frequency & Percent & $\begin{array}{c}\text { Valid } \\
\text { Percent }\end{array}$ & $\begin{array}{c}\text { Cumulative } \\
\text { Percent }\end{array}$ \\
\hline \multirow{8}{*}{ Valid } & 1.0 & 2 & 6.9 & 6.9 & 6.9 \\
\hline & 2.0 & 8 & 27.6 & 27.6 & 34.5 \\
\hline & 3.0 & 7 & 24.1 & 24.1 & 58.6 \\
\hline & 4.0 & 7 & 24.1 & 24.1 & 82.8 \\
\hline & 5.0 & 3 & 10.3 & 10.3 & 93.1 \\
\hline & 7.3 & 1 & 3.4 & 3.4 & 96.6 \\
\hline & 9.0 & 1 & 3.4 & 3.4 & 100.0 \\
\hline & Total & 29 & 100.0 & 100.0 & \\
\hline
\end{tabular}

\begin{tabular}{|c|c|c|c|c|c|}
\hline \multicolumn{6}{|c|}{ QUANTITY IN POCKETS } \\
\hline & & Frequency & Percent & $\begin{array}{c}\text { Valid } \\
\text { Percent }\end{array}$ & $\begin{array}{c}\text { Cumulative } \\
\text { Percent }\end{array}$ \\
\hline \multirow{7}{*}{ Valid } & .25 & 1 & 3.4 & 3.4 & 3.4 \\
\hline & .50 & 10 & 34.5 & 34.5 & 37.9 \\
\hline & 1.00 & 14 & 48.3 & 48.3 & 86.2 \\
\hline & 2.00 & 2 & 6.9 & 6.9 & 93.1 \\
\hline & 3.00 & 1 & 3.4 & 3.4 & 96.6 \\
\hline & 4.00 & 1 & 3.4 & 3.4 & 100.0 \\
\hline & Tota & 29 & 100.0 & 100.0 & \\
\hline
\end{tabular}

\begin{tabular}{|c|c|c|c|c|c|}
\hline \multicolumn{2}{|l|}{ VOMITING } & Frequency & Percent & $\begin{array}{c}\text { Valid } \\
\text { Percent }\end{array}$ & $\begin{array}{c}\text { Cumulative } \\
\text { Percent }\end{array}$ \\
\hline \multirow{4}{*}{ Valid } & No & 2 & 6.9 & 6.9 & 6.9 \\
\cline { 2 - 6 } & NO & 21 & 72.4 & 72.4 & 79.3 \\
\cline { 2 - 6 } & Yes & 6 & 20.7 & 20.7 & 100.0 \\
\cline { 2 - 6 } & Total & 29 & 100.0 & 100.0 & \\
\hline
\end{tabular}

\begin{tabular}{|l|c|c|c|c|c|}
\hline \multicolumn{2}{|l|}{ RECOVERY } & Frequency & Percent & $\begin{array}{c}\text { Valid } \\
\text { Percent }\end{array}$ & $\begin{array}{c}\text { Cumulative } \\
\text { Percent }\end{array}$ \\
\hline Valid & Recovered & 29 & 100.0 & 100.0 & 100.0 \\
\hline
\end{tabular}

\begin{tabular}{|l|c|c|c|c|c|}
\hline \multicolumn{3}{|l|}{ SEQULAE } & & \\
\hline & Frequency & Percent & $\begin{array}{c}\text { Valid } \\
\text { Percent }\end{array}$ & $\begin{array}{c}\text { Cumulative } \\
\text { Percent }\end{array}$ \\
\hline Valid & NIL & 29 & 100.0 & 100.0 & 100.0 \\
\hline
\end{tabular}

\begin{tabular}{|c|c|c|c|c|c|}
\hline \multicolumn{6}{|c|}{ FREQUENCY OF VOMITING } \\
\hline \multirow{2}{*}{} & Frequency & Percent & $\begin{array}{c}\text { Valid } \\
\text { Percent }\end{array}$ & $\begin{array}{c}\text { Cumulative } \\
\text { Percent }\end{array}$ \\
\hline \multirow{5}{*}{ Valid } & .0 & 23 & 79.3 & 79.3 & 79.3 \\
\cline { 2 - 6 } & 1.0 & 3 & 10.3 & 10.3 & 89.7 \\
\cline { 2 - 6 } & 2.0 & 1 & 3.4 & 3.4 & 93.1 \\
\cline { 2 - 6 } & 5.0 & 1 & 3.4 & 3.4 & 96.6 \\
\cline { 2 - 6 } & 7.0 & 1 & 3.4 & 3.4 & 100.0 \\
\cline { 2 - 6 } & Total & 29 & 100.0 & 100.0 & \\
\hline
\end{tabular}

\begin{tabular}{|l|c|c|c|c|c|}
\hline \multicolumn{2}{|l|}{ STOMACH WASH } & Frequency & Percent & $\begin{array}{c}\text { Valid } \\
\text { Percent }\end{array}$ & $\begin{array}{c}\text { Cumulative } \\
\text { Percent }\end{array}$ \\
\hline Valid & Yes & 29 & 100.0 & 100.0 & 100.0 \\
\hline
\end{tabular}
\begin{tabular}{|l|c|c|c|c|}
\hline WIHT WATER & Frequency & Percent & $\begin{array}{c}\text { Valid } \\
\text { Percent }\end{array}$ & $\begin{array}{c}\text { Cumulative } \\
\text { Percent }\end{array}$ \\
\hline
\end{tabular}


WITH SALINE

\begin{tabular}{|c|l|c|c|c|c|}
\hline \multicolumn{2}{|c|}{} & Frequency & Percent & $\begin{array}{c}\text { Valid } \\
\text { Percent }\end{array}$ & $\begin{array}{c}\text { Cumulative } \\
\text { Percent }\end{array}$ \\
\hline \multirow{3}{*}{ Valid } & No & 26 & 89.7 & 89.7 & 89.7 \\
\cline { 2 - 6 } & Yes & 3 & 10.3 & 10.3 & 100.0 \\
\cline { 2 - 6 } & Total & 29 & 100.0 & 100.0 & \\
\hline
\end{tabular}

\begin{tabular}{|l|l|c|c|c|c|}
\hline \multicolumn{2}{|l|}{ RTA } & Frequency & Percent & $\begin{array}{c}\text { Valid } \\
\text { Percent }\end{array}$ & $\begin{array}{c}\text { Cumulative } \\
\text { Percent }\end{array}$ \\
\hline \multirow{3}{*}{ Valid } & No & 24 & 82.8 & 82.8 & 82.8 \\
\cline { 2 - 6 } & Yes & 5 & 17.2 & 17.2 & 100.0 \\
\cline { 2 - 6 } & Total & 29 & 100.0 & 100.0 & \\
\hline
\end{tabular}

\begin{tabular}{|c|l|c|c|c|c|}
\hline \multicolumn{2}{|l|}{ ACTIVE CHARCOL } \\
\hline \multirow{2}{|c|}{} & Frequency & Percent & $\begin{array}{c}\text { Valid } \\
\text { Percent }\end{array}$ & $\begin{array}{c}\text { Cumulative } \\
\text { Percent }\end{array}$ \\
\hline \multirow{3}{*}{ Valid } & No & 26 & 89.7 & 89.7 & 89.7 \\
\cline { 2 - 6 } & Yes & 3 & 10.3 & 10.3 & 100.0 \\
\cline { 2 - 6 } & Total & 29 & 100.0 & 100.0 & \\
\hline
\end{tabular}

\begin{tabular}{|c|c|c|c|c|c|}
\hline \multicolumn{2}{|c|}{ ANTE EMITIC } & Frequency & Percent & $\begin{array}{c}\text { Valid } \\
\text { Percent }\end{array}$ & $\begin{array}{c}\text { Cumulative } \\
\text { Percent }\end{array}$ \\
\hline \multirow{3}{*}{ Valid } & No & 23 & 79.3 & 79.3 & 79.3 \\
\cline { 2 - 6 } & Yes & 6 & 20.7 & 20.7 & 100.0 \\
\cline { 2 - 6 } & Total & 29 & 100.0 & 100.0 & \\
\hline
\end{tabular}

\begin{tabular}{|l|l|c|c|c|c|}
\hline \multicolumn{2}{|l|}{ PPI } & Frequency & Percent & $\begin{array}{c}\text { Valid } \\
\text { Percent }\end{array}$ & $\begin{array}{c}\text { Cumulative } \\
\text { Percent }\end{array}$ \\
\hline \multirow{3}{*}{ Valid } & No & 23 & 79.3 & 79.3 & 79.3 \\
\cline { 2 - 6 } & Yes & 6 & 20.7 & 20.7 & 100.0 \\
\cline { 2 - 6 } & Total & 29 & 100.0 & 100.0 & \\
\hline
\end{tabular}

\begin{tabular}{|l|c|c|c|c|c|}
\hline \multicolumn{2}{|l|}{ VIT-K INJ } & \multicolumn{4}{l|}{} \\
\hline \multicolumn{2}{|l|}{} & Frequency & Percent & $\begin{array}{c}\text { Valid } \\
\text { Percent }\end{array}$ & $\begin{array}{c}\text { Cumulative } \\
\text { Percent }\end{array}$ \\
\hline Valid & No & 29 & 100.0 & 100.0 & 100.0 \\
\hline
\end{tabular}

\begin{tabular}{|l|l|c|c|c|c|}
\hline \multicolumn{2}{|l|}{ IV FLUIDS } & Frequency & Percent & $\begin{array}{r}\text { Valid } \\
\text { Percent }\end{array}$ & $\begin{array}{c}\text { Cumulative } \\
\text { Percent }\end{array}$ \\
\hline \multirow{3}{*}{ Valid } & iNj.atropiN & 1 & 3.4 & 3.4 & 3.4 \\
\cline { 2 - 6 } & No & 28 & 96.6 & 96.6 & 100.0 \\
\cline { 2 - 6 } & Total & 29 & 100.0 & 100.0 & \\
\hline
\end{tabular}

\begin{tabular}{|c|l|c|c|c|c|}
\hline \multicolumn{2}{|l|}{ AGE GROUP } & Frequency & Percent & $\begin{array}{c}\text { Valid } \\
\text { Percent }\end{array}$ & $\begin{array}{c}\text { Cumulative } \\
\text { Percent }\end{array}$ \\
\hline \multirow{4}{*}{ Valid } & $15-20$ & 8 & 27.6 & 27.6 & 27.6 \\
\cline { 2 - 6 } & $21-30$ & 13 & 44.8 & 44.8 & 72.4 \\
\cline { 2 - 6 } & $31-40$ & 6 & 20.7 & 20.7 & 93.1 \\
\cline { 2 - 6 } & $41-50$ & 2 & 6.9 & 6.9 & 100.0 \\
\cline { 2 - 6 } & Total & 29 & 100.0 & 100.0 & \\
\hline
\end{tabular}

\begin{tabular}{|c|c|c|c|c|c|}
\hline \multicolumn{2}{|l|}{ AGE GROUP } & Frequency & Percent & $\begin{array}{r}\text { Valid } \\
\text { Percent }\end{array}$ & $\begin{array}{c}\text { Cumulative } \\
\text { Percent }\end{array}$ \\
\hline \multirow{5}{*}{ Valid } & $15-20$ & 8 & 27.6 & 27.6 & 27.6 \\
\cline { 2 - 6 } & $21-30$ & 13 & 44.8 & 44.8 & 72.4 \\
\cline { 2 - 6 } & $31-40$ & 6 & 20.7 & 20.7 & 93.1 \\
\cline { 2 - 6 } & $41-50$ & 2 & 6.9 & 6.9 & 100.0 \\
\cline { 2 - 6 } & Total & 29 & 100.0 & 100.0 & \\
\hline
\end{tabular}

Descriptives

Descriptive Statistics

\begin{tabular}{|l|c|c|c|c|c|}
\hline & $\mathrm{N}$ & Minimum & Maximum & Mean & $\begin{array}{c}\text { Std. } \\
\text { Deviation }\end{array}$ \\
\hline $\begin{array}{l}\text { QUANTITY IN } \\
\text { POCKETS }\end{array}$ & 29 & .25 & 4.00 & 1.0431 & .80198 \\
\hline $\begin{array}{l}\text { FREQUENCY } \\
\text { OF VOMITING }\end{array}$ & 29 & .0 & 7.0 & .586 & 1.5928 \\
\hline DURATION & 29 & .0 & .0 & .000 & .0000 \\
\hline $\begin{array}{l}\text { NO. OF DAYS } \\
\text { STAY 29 }\end{array}$ & 1.0 & 9.0 & 3.390 & 1.7432 \\
\hline Valid N (listwise) & 29 & & & & \\
\hline
\end{tabular}

\begin{tabular}{|c|c|c|c|c|c|c|c|c|}
\hline \multicolumn{9}{|c|}{ SEX * QUANTITY IN POCKETS Crosstabulation } \\
\hline \multicolumn{9}{|c|}{ Count } \\
\hline & & \multicolumn{6}{|c|}{ QUANTITY IN POCKETS } & \multirow[t]{2}{*}{ Total } \\
\hline & & .25 & .50 & 1.00 & 2.00 & 3.00 & 4.00 & \\
\hline \multirow{2}{*}{ SEX } & FEMALE & 1 & 6 & 7 & 1 & 0 & 1 & 16 \\
\hline & MALE & 0 & 4 & 7 & 1 & 1 & 0 & 13 \\
\hline \multicolumn{2}{|l|}{ Total } & 1 & 10 & 14 & 2 & 1 & 1 & 29 \\
\hline
\end{tabular}

\begin{tabular}{|c|c|c|c|c|}
\hline \multicolumn{5}{|c|}{ SEX * EPIPAIN Crosstabulation } \\
\hline \multicolumn{5}{|c|}{ Count } \\
\hline & & \multicolumn{2}{|c|}{ EPIPAIN } & \multirow[t]{2}{*}{ Total } \\
\hline & & No & Yes & \\
\hline \multirow{2}{*}{ SEX } & FEMALE & 14 & 2 & 16 \\
\hline & MALE & 12 & 1 & 13 \\
\hline \multicolumn{2}{|l|}{ Total } & 26 & 3 & 29 \\
\hline
\end{tabular}

Sex $=$ Female

\begin{tabular}{|l|c|c|c|c|c|}
\hline Descriptive Statistics $^{\mathrm{a}}$ & $\mathrm{N}$ & Minimum & Maximum & Mean & $\begin{array}{c}\text { Std. } \\
\text { Deviation }\end{array}$ \\
\hline $\begin{array}{l}\text { QUANTITY IN } \\
\text { POCKETS }\end{array}$ & 16 & .25 & 4.00 & 1.0156 & .89661 \\
\hline $\begin{array}{l}\text { FREQUENCY OF } \\
\text { VOMITING }\end{array}$ & 16 & .0 & 7.0 & .875 & 2.0616 \\
\hline DURATION & 16 & .0 & .0 & .000 & .0000 \\
\hline $\begin{array}{l}\text { NO. OF DAYS } \\
\text { STAY }\end{array}$ & 16 & 1.0 & 9.0 & 3.456 & 2.2235 \\
\hline Valid N (listwise) & 16 & & & & \\
\hline a. SEX = FEMALE & & & & \\
\hline SEX = MALE
\end{tabular}


Descriptive Statistics $^{\mathrm{a}}$

\begin{tabular}{|l|c|c|c|c|c|}
\hline & $\mathrm{N}$ & Minimum & Maximum & Mean & $\begin{array}{c}\text { Std. } \\
\text { Deviation }\end{array}$ \\
\hline $\begin{array}{l}\text { QUANTITY IN } \\
\text { POCKETS }\end{array}$ & 13 & .50 & 3.00 & 1.0769 & .70256 \\
\hline $\begin{array}{l}\text { FREQUENCY } \\
\text { OF VOMITING }\end{array}$ & 13 & .0 & 2.0 & .231 & .5991 \\
\hline DURATION & 13 & .0 & .0 & .000 & .0000 \\
\hline $\begin{array}{l}\text { NO. OF DAYS } \\
\text { STAY }\end{array}$ & 2.0 & 5.0 & 3.308 & .9473 \\
\hline Valid N (listwise) & 13 & & & & \\
\hline a. SEX = MALE & & & & \\
\hline
\end{tabular}

\section{Results}

All reported cases were found to be suicidal poisoning. Majority of cases were in the age group of 16 yrs-50 yrs, Female (16,55.2\%) out numbers males, $(13,44.8 \%)$, student attempted to commit suicide much less than others. House wives and daily wages labourers. The poison consumed was yellow cow dung powder poison. In our study the mortality was nil.

\section{Conclusion}

This study adds information to the existing data which may help to develop prevention strategies. Health education to the house wives and coolies and student by either by house trained faculty or physiologists and early detection of risk taking behavior in adults to some extent to prevent self harming in all patients. Ban on cow dung powder sales in grocery shops should be followed by District Authority.

\section{References}

1. Hisham MD, Vijayakumar A, Rajesh N, Sivakumar MN. Auramine-o and malachite green poisoning: Rare and fatal. Indian $\mathbf{J}$ Pharm Pract 2013;6:72-4.

2. Singh UK, Layland FC, Prasad R, Singh S. Miscellaneous: Poisoning in Children. $3 \mathrm{rd}$ ed. New Delhi: Jaypee Publishers; 2006. p. 213-15.

3. Karikalan T, Murugan M. Profile of poisoning case in a tertiary care hospital,
Tamil Nadu. J Evol Med Dent Sci 2014;3:12754-60.

4. Krishnamoorthy A, Subramanian R, Dhanaselvi P, Prabhu RS, Jayanthi V. Clinical presentation of cow dung powder poison - A preliminary communication jou Assoc Physicians India 2001;49.

5. Angulo P. Use of ursodeoxycholic acid in patients with liver disease. Curr Gastroenterol Rep 2002;4:37-44.

6. Parodi S, Santi L, Russo P, Albini A, Vecchio $\mathrm{D}$, Pala $\mathrm{M}$, et al. DNA damage induced by Auramine $\mathrm{O}$ in liver, kidney, and bone marrow of rats and mice,and in a human cell line (Alkaline elution assay and SCE induction). J Toxicol Environ Health 1982; 9:941-52. [PUBMED]

7. [IARC. Monographs on the Evaluation of the Carcinogenic Risk of Chemicals to Humans. Geneva: World Health Organization, International Agency for Research on Cancer, 1972-PRESENT. work). Available at: http://monographs.iarc.fr/ENG/Classification/ index .php p. S7 118 (1987)] **Peer Reviewed

8. Sherfudeen KM, Kaliannan SK, Dammalapati PK.Cow dung powder poisoning. Indian J Crit Care Med2015; 19:684http://www.ijccm.org/text.asp?2015/19 /11/684/169357. /Auramine/ [Parodi S Et Al; J Toxicol Environ Health 9 (5-6): 941 (Prof. Dr. Edwin joe.M.D.B.L Director of Medical Education ,Govt of Tamilnadu 1982)]

9. Journal of Evolution of medical and Dental science JEMDS. tkarigalan, Mmurugan, profile of poisoning cases in tertian care hospital. Tamil nadu .20014; vol-3, issue 56, october 27;page 12754, 12760,v ol:10.14260/Jemds/2014,3692.

10. Cow dung powder poisoning: Khaja Mohideen Sherfudeen, and Pavan kum additional article informationar. Dammalapatti. 